Proceedings of the XXIII Conference on Applied Crystallography, Krynica Zdrój, Poland, September 20-24, 2015

\title{
Dendrite Growth in Selector-Root Area of Single Crystal CMSX-4 Turbine Blades
}

\author{
W. Bogdanowicz ${ }^{a}$, A. Tondos ${ }^{a, *}$, J. KraWczyK $^{a}$, R. Albrecht ${ }^{a}$ And J. Sieniawski ${ }^{b}$ \\ ${ }^{a}$ University of Silesia, Institute of Materials Science, 75 Pułku Piechoty 1A, 41-500 Chorzów, Poland \\ ${ }^{b}$ Rzeszów University of Technology, Department of Materials Science, W. Pola 2, 35-959 Rzeszów, Poland
}

\begin{abstract}
The single crystal turbine blades made of CMSX-4 nickel-based superalloy were studied. The turbine blades were obtained by the Bridgman technique with withdrawal rate of $5 \mathrm{~mm} / \mathrm{min}$. The samples, cut-off from root part of blades and containing the fragment of the selector, were studied. The effect of selector geometry on the dendrites growth and defects formation in the selector-root area of the blade were analyzed. The Laue diffraction, scanning electron microscopy, and X-ray diffraction topography were applied. It was found that, during crystallization of the selector, the dendrite cores, after reaching the surface of mould, may bend, if the angle between dendrite cores and the mould surface was equal to $12^{\circ}$. When the angle was equal to $24^{\circ}$ the growth of dendrites has been stopped. It can be stated that the defects, which appeared in the selector were inherited by the root part.
\end{abstract}

DOI: 10.12693/APhysPolA.130.1107

PACS/topics: 61.66.Dk, 61.72.--y, 61.10.Nz

\section{Introduction}

Nowadays single crystal nickel-based superalloys are adapted to work in extreme conditions, especially in aircraft engine and stationary gas turbine. The unusual ability to retain combination of mechanical properties and corrosion resistance at high temperatures [1] make those materials useful for producing the blades for high temperature parts in high pressure turbines (HPT). The blades with complex shape are difficult to obtain and tend to create many growth defects during solidification. Those defects affect the blade causing decrease of its mechanical properties. Hence, single crystal turbine blades may possess critical areas with regard to the crystallization process. The most affected area is situated between root and selector (area A, Fig. 1 (left)). Considering
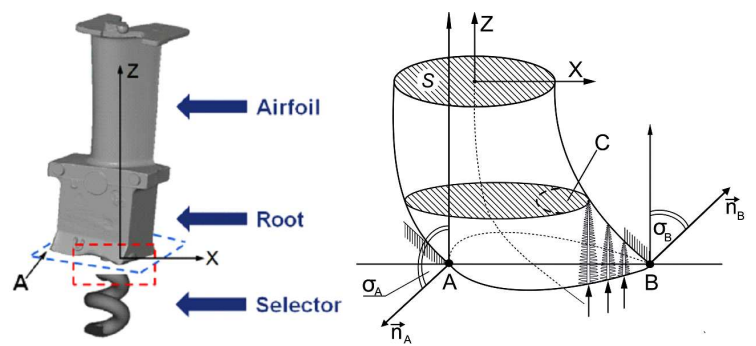

Fig. 1. (left) Scheme of typical turbine blade with marked main parts and critical area $A$. $Z$ - withdrawal axis. (right) Geometry of spiral selector part which is connected to the root by surface $S$.

the fact that during process of crystallization the spiral selector provides the orientation for the whole casting element [2], the selector geometry and its connection with the root have a significant influence on the way of the

\footnotetext{
* corresponding author; e-mail: atondos@us.edu.pl
}

dendrites growth. Therefore, the influence of selector geometry and root areas located near the selector on crystallization process was investigated. The location and mechanism of defects formation were investigated as well. Figure 2 presents fragment of the selector with some geometrical parameters, marked as $\sigma_{A}$ and $\sigma_{B}$ angles, which describe the inclination of vectors $n_{A}$ and $n_{B}$ (perpendicular to the selector walls), relative to the withdrawal direction $(Z)$. The angles $\sigma_{A}$ and $\sigma_{B}$ are determined at the points $\mathrm{A}$ and $\mathrm{B}$ of plane parallel to the section $\mathrm{S}$, which is perpendicular to the axis $Z$ (Fig. 1 (right)).

The influence of selector geometry on the dendrites growth and defects creation was studied by two X-ray diffraction methods: Laue and X-ray topography. Additionally, the dendrite structure was analyzed by scanning electron microscopy (SEM).

\section{Experimental}

Single crystal blades of CMSX-4 nickel based superalloy were produced by the Bridgman technique with the withdrawal rate of $5 \mathrm{~mm} / \mathrm{min}$ using an ALD Vacuum Technologies furnace in Rzeszów University of Technology. Microstructure of CMSX-4 superalloys consist mainly of $\gamma$ phase (matrix) strengthened by reinforcing $\gamma^{\prime}$ phase $\left(\mathrm{Ni}_{3} \mathrm{Al}\right)$ with high volume fraction - up to $70 \%$ [3]. The $\gamma^{\prime}$ phase is highly coherent with the $\gamma$ phase. Those two phases have a small mismatch between lattice parameters [4]. The samples for investigation were cutoff perpendicular and parallel to the withdrawal axis $Z$ (Fig. 1) from root part of turbine blades with fragment of selector (Fig. 2), which will be further called selectorroot (SR) area. Surface of the samples were prepared using the standard for superalloys standard metallographic procedure [5].

The crystallographic orientation for all samples was defined by the Laue method. The X-ray topograms were obtained with the use of the PANalytical microfocus X-ray source equipped with the Auleytner camera [6]. The reflexes of $\mathrm{Cu} K_{\alpha}$ radiation were received on X-ray film, 


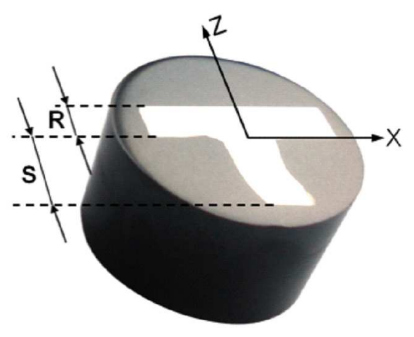

Fig. 2. Longitudinal metallographic section of samples prepared from selector-root area. $R$ and $S$ - fragments of the root and selector respectively.

which oscillate coupled with sample. SEM was used to study the structural defects and the dendrites arrangement in blades. Connection of almost forty micro-images from SEM allowed to visualize the dendrite structure of whole surface of samples (so-called SEM macro-image).

\section{Results and discussion}

SEM macro-image of longitudinal section of SR area (Fig. 3) shows several details of sample microstructure, which represent the structural defects forming during different processes of dendrites growth over crystallization. For the selector it can be observed that $\sigma_{B}$ angle has significant influence (Fig. 1 (right)). Depending on the value of $\sigma_{B}$ angle, the dendrites can be bent or being stopped on the mould wall surrounding the selector. It is possible to reveal three different options of dendrite growth (Fig. 4). First of them, appears when the $\sigma_{B}$ value is $90^{\circ}$. The dendrite grows parallel to the surface of the mould and none effects are observed. In the case of $\sigma_{B}$ angle value is higher or equal $24^{\circ}$ and the dendrite growth intermits, when the core reaches the mould wall. However, if the $\sigma_{B}$ value is $12^{\circ}$ or less, the dendrites can bend along the surface of the mould. Figure $3 \mathrm{~b}$ shows the way of dendrite bending. At the beginning the dendrite grows parallel to $Z$ axis, along the $\mathrm{N}_{1}$ direction, but when the dendrite encounters mould surface (in $\mathrm{K}$ point) it begins to bend along the direction $\mathrm{N}_{2}$. At the end of the process the dendrite grows parallel to $\mathrm{N}_{3}$ direction, that is parallel to $Z$ axis again. It follows that the dendrite bending may occur, creating structural defects in the further crystallization stage. Hence, it may be concluded that the selector geometry affects the growth of dendrites, which can cause creation of defects in the root section, while bending on the mould wall. Additionally this bending may cause some changes in neighboring dendrite structure (areas CC, Fig. 3b). Change of dendrite growth direction should change the crystal orientation.

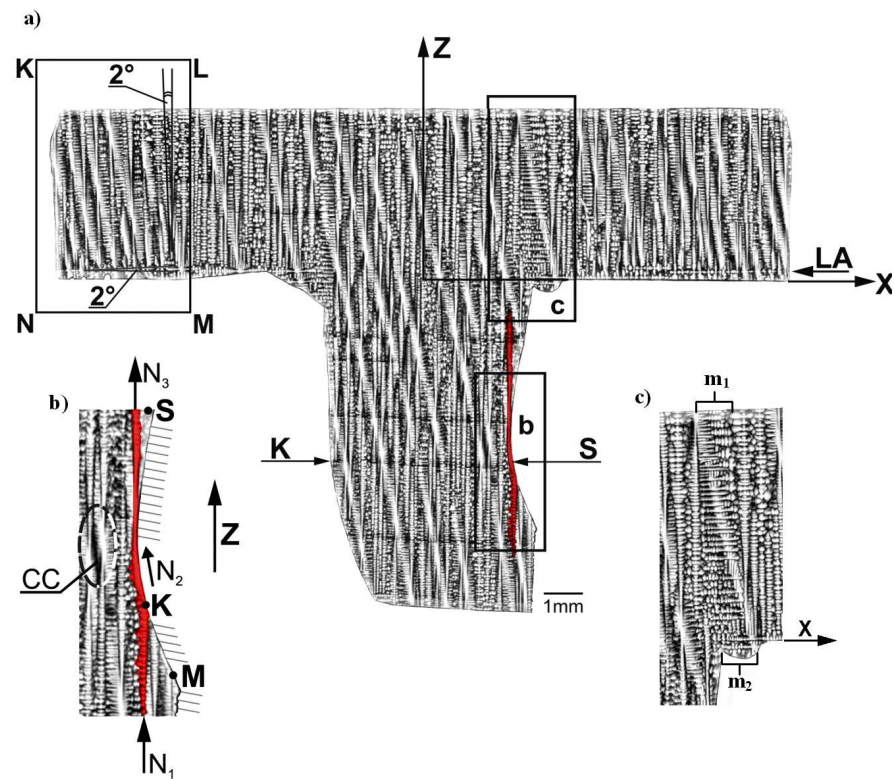

Fig. 3. Typical SEM macro image of dendrite structure, visualized on the sample surface - longitudinal section of $\mathrm{SR}$ area (a) with some magnified parts (b,c). Z — blade axis, parallel to the crystallization direction. MKS — surface of the mould.

In Fig. 3c it can be observed that in the root area there is an increased concentration of secondary dendrite arms (strip between $m_{1}$ and $m_{2}$ ) in the place, where the crystallization front was extended by beginning of crystallization along axis $X$.

In the area marked by KLMN frame in Fig. 3a it can be observed that the dendrites are inclined about 2 degrees to axis $Z$. Also it may be observed that the long secondary dendrite arms (for example LA) are inclined at 2 degrees to axis $X$.

Figure 5 presents the X-ray diffraction topogram which was obtained from the surface in the same way as for macro SEM images presented in Fig. 3a. The X-ray topogram consists of contrast bands with different intensity 

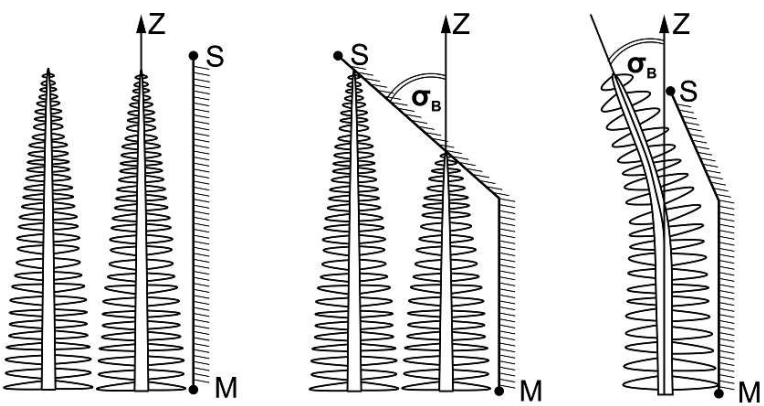

Fig. 4. Different options of dendrites growth depending on angle $\sigma_{B}$ value. $M S$ - surface of the mould.

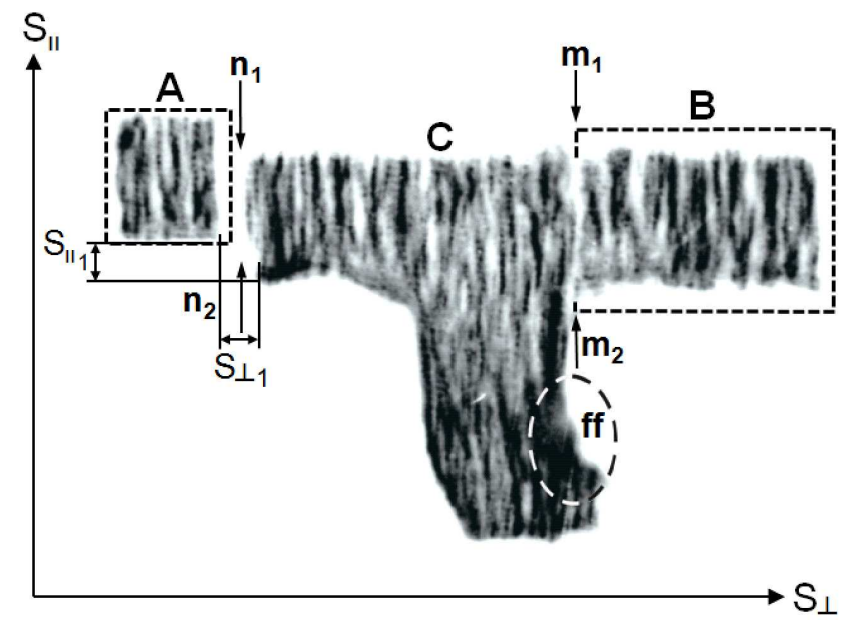

Fig. 5. Typical X-ray reflective topogram of longitudinal section of SR area. 113 reflection. $\mathrm{Cu} K_{\alpha}$ radiation. Coupled film and sample oscillation. $n_{1}-n_{2}, m_{1}-m_{2}$ - low angle boundaries, $A, B, C$ - subgrains, $S_{\|}$axis of sample oscillation, which is parallel to $Z$ axis of blade.

which were arranged along axis $Z$ and almost in the same direction to the primary dendrite arms as shown in Fig. 3a. The dendritic structure may include the strain region appearing on topogram as area with blurred contrast (area $f f$, Fig. 5). This effect was created in the place where the dendrites cores reach the mould wall. The blurred contrast may suggest a macro-scale local deformation. Dendrite bending visible in the SEM image (Fig. 3b) may be the source of darker contrast on the topogram and consequently lead to low angle boundaries formation $\left(m_{1}-m_{2}\right.$ in Fig. 5), which was observed additionally on the microstructure as area of secondary dendrites arms elongated in direction $X$ (Fig. 3c). Low angle boundaries (Fig. $5, n_{1}-n_{2}, m_{1}-m_{2}$ ) presented in the topogram are passes through the entire area of the root, dividing the sample into three subgrains (A, B, C). Image of subgrain A on the topogram is shifted in two directions: $S_{\perp}$ and $S_{\|}$. The angle of misorientation calculated from values $S_{\perp}$ and $S_{\|}$, according to the method presented in paper [6] is close to $2^{\circ}$. This value can be compared with the dendrites inclination, visualized in Fig. 3a. The boundary between part $\mathrm{B}$ and $\mathrm{C}$ is not clearly visible on microstructure, due to the arcminutes value of misorientation. The boundary $m_{1}-m_{2}$ (Fig. 5) is created in the place where the crystallization front begun to extend.

To verify the area of selector, where probably the strain regions are formed, the cross-section by plane KS of selector (Fig. 3a) was prepared. As shown in Fig. 6 most of the topogram area presents the various contrast from dendrite arms arrangement. However, the area $\mathrm{C}$ shows the diffusion contrast. This is the place where growing dendrite reaches the mould wall.

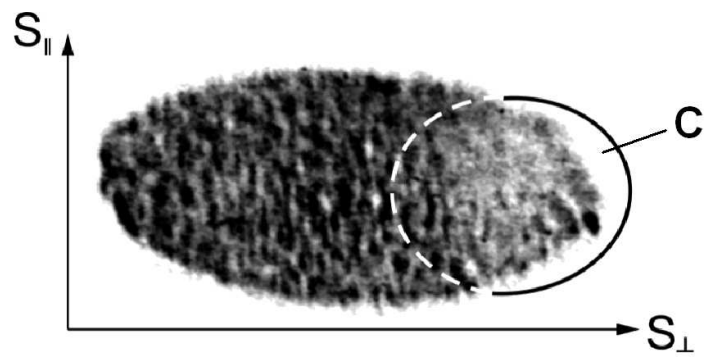

Fig. 6. X-ray topogram of selector cross-section. 002 reflection of $\mathrm{Cu} K_{\alpha}$ radiation. Coupled film and sample oscillation. $S_{\|}$- axis of sample oscillation. $S_{\perp}$ - axis parallel to the axis $X$ (Fig. 3a).

\section{Conclusions}

The selector geometry can influence on the growth of the dendrites, which can bend during crystallization process. The bend process may cause: changes in the crystal orientation and misorientation of the neighboring dendrites near the selector wall; facilitating of secondary dendrite arms growth and subgrains forming in the root. The strain region is formed in the place, where the dendrite growth is confined by the surface of the mould. The low angle boundaries created in the root may be the consequences of facilitated growth of the secondary dendrite arms in the root area. The defects which appear in the selector part are probably inherited by the root part. $\mathrm{p}$

\section{Acknowledgments}

The research was financed by the Polish National Centre for Research and Development (NCBiR) under Grant no. INOTECH-K2/IN2/57/182858/NCBR/13.

\section{References}

[1] H.J. Dai, N. D'Souza, H.B. Dong, Metall. Mater. Trans. A 42, 3430 (2011).

[2] J.R. Davis, Heat-Resistant Materials, ASM International, Materials Park (OH) 1997.

[3] W. Bogdanowicz, Scr. Mater. 37, 829 (1997).

[4] R.C. Reed, The Superalloys. Fundamentals and Applications, Cambridge University Press, Cambridge 2006.

[5] D.C. Zipperian, Metallographic Handbook, Chief Technical Officier PACE Technologies, Tucson (AR) 2011.

[6] W. Bogdanowicz, R. Albrecht, A. Onyszko, J. Sieniawski, Solid State Phenom. 203-204, 63 (2013). 